\title{
Demonstration of the Validity of the Early Warning in Online Monitoring System for Nuclear Power Plants
}

\author{
Ji Ho Min ${ }^{1}$, Dae-Woong Kim², Chi-Yong Park ${ }^{3}$ \\ ${ }^{1}$ Assistant Research Engineer, Central Research Institute, KHNP, Korea \\ ${ }^{2}$ Principal Research Engineer, Central Research Institute, KHNP, Korea \\ ${ }^{3}$ Group Leader, Central Research Institute, KHNP, Korea
}

\begin{abstract}
This paper presents the validity and usefulness of early warning in online monitoring system for nuclear power plants. Early warning is one of the core functions of the online monitoring system, which uses pattern recognition to predict and alert potential problems in the equipment or system. This function was developed by using the AAKR technique and has been operated since 2016. We show that the early warning system is operating properly through an analysis of the operation result of the system, and present two cases that represent the role and reliability of the system. In the first case, the system detected a failure of measuring instrument in advance. And the other case, the system went through pattern relearning to overcome environmental changes.
\end{abstract}

\section{Introduction}

Nuclear power plants are complex facilities that combine various equipments and systems. Fault or malfunction of single equipment can turn into accident which affects the entire plant. Conventionally, the monitoring and diagnosis of equipments have been carried out individually by each power plant, but with the development of technology, there are growing demands for integrated monitoring and diagnosis to enhance safety (E. Davis et al., 2000; B. Rasmussen et al., 2004). To meet these demands, Korea Hydro \& Nuclear Power Co., Ltd. (KHNP) has established and operated an online monitoring system since 2016, which can monitor and diagnose 24 nuclear power plants in real time. Early warning is one of the key functions of this system. It uses pattern learning and artificial intelligence to detect a slight fault symptom of equipments in advance and lets the operators prevent or prepare for failure and minimize losses caused by unplanned maintenance.

In this paper, we introduce the online monitoring system and its early warning, which is one of the main functions, and describe the validity and usefulness of early warning through case studies.

\section{Online Monitoring System}

KHNP's online monitoring system collects, monitors and analyzes the signals from the 24 nuclear power plants in real time. This system ensures the safety and efficiency of the nuclear power plants through centralized monitoring; detects any abnormalities of the equipment and systems in advance and generates early warning; and provides diagnostic tool for expert analysis. 


\subsection{System configuration}

A schematic diagram of the system is shown in Figure 1. A local server is installed at each four sites of the power plants, and real-time operating data is collected from the plant monitoring system of the power plants and transmitted to the central server in the headquarters. The central server monitors and analyzes the operating data and sends the results to the headquarters and the central research institute in real time. The results analyzed by the system are further scrutinized and validated by experts, and when validated, the plants are notified to take action.

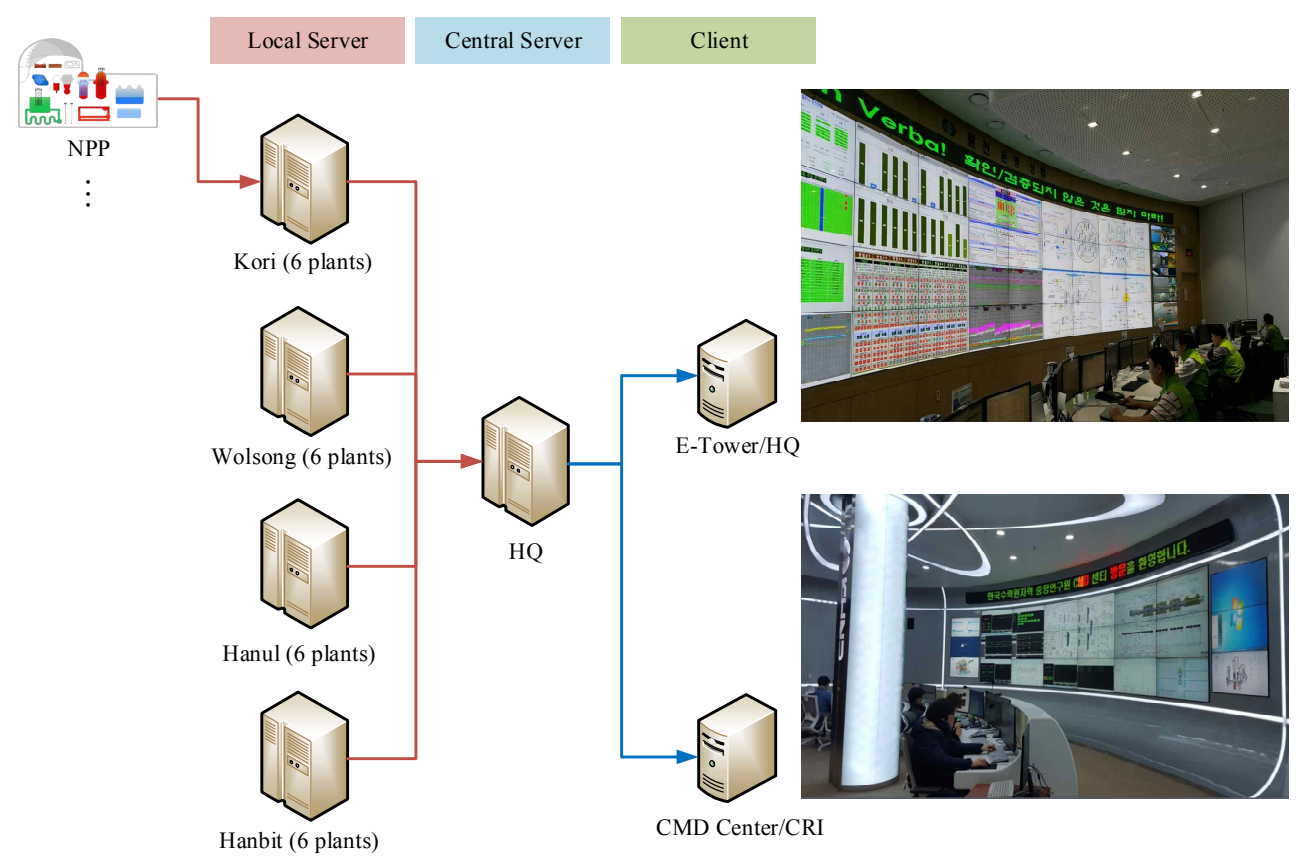

Figure 1. Configuration of Online Monitoring System

\subsection{Principles of early warnings}

In most cases, a failure of equipment or systems starts from a microscopic defect, which may develop into a serious failure. Any unusual symptoms can be observed during the growth of a failure. As shown in Figure 2, an early warning system is a technology to prevent or prepare against malfunction of the systems by detecting and alerting abnormal symptoms in advance, thus improving safety and operational efficiency of the power plant (Q. Zhang et al., 1994).

There are generally parametric and nonparametric methods for data-driven models that can predict the current state compared with the previous ones in its normal operations. The parametric method can be applied based on the assumption that the data distribution is known. In contrast, the nonparametric method is a method of estimating the current state without any information about the data distribution and the kernel regression method is a representative one (G. Kauermann et al., 1998; K.W. Hines et al., 2008). 


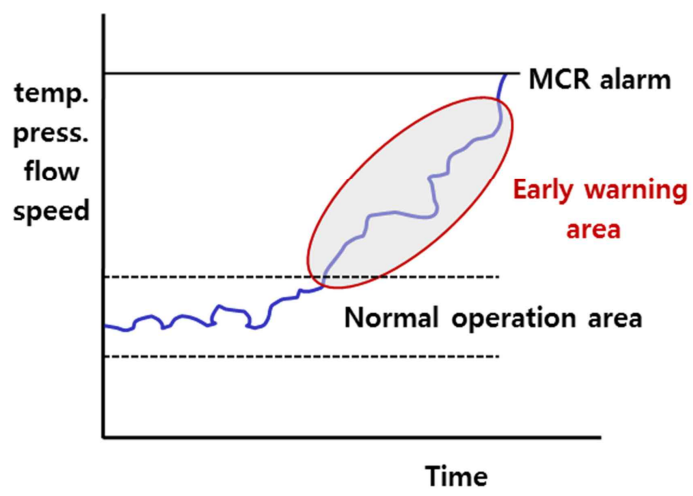

Figure 2. Concept of Early Warning

In this system, AAKR(Auto-Associative Kernel Regression) technique is applied, which is the nonparametric, empirical modeling method.

The AAKR technique estimates the expected variables $\widehat{X}$ by comparing the currently measured variables $\mathrm{X}$ with the past ones $\mathrm{X}^{n c}$ collected during normal operation. In order to apply the AAKR technique, the memory vector $\mathrm{X}^{n c}$ must be generated from the past normal operating data. When the number of measurement variables is $\mathrm{J}$ and the number of data is $\mathrm{N}, \mathrm{X}^{n c}$ is the matrix of $\mathrm{N} \times \mathrm{J}$ as follows:

$$
\mathrm{X}^{\mathrm{nc}}=\left[\begin{array}{cccc}
\mathrm{x}_{1,1} & \mathrm{x}_{1,2} & \cdots & \mathrm{x}_{1, \mathrm{~J}} \\
\mathrm{x}_{2,1} & \mathrm{x}_{2,2} & \vdots & \mathrm{x}_{2, \mathrm{~J}} \\
\vdots & \vdots & \ddots & \vdots \\
\mathrm{x}_{\mathrm{N}, 1} & \mathrm{x}_{\mathrm{N}, 2} & \cdots & \mathrm{x}_{\mathrm{N}, \mathrm{J}}
\end{array}\right]
$$

When a vector $\mathrm{X}=\left[\begin{array}{llll}x_{1} & x_{2} & \ldots & x_{J}\end{array}\right]$ composed of currently measured variables is inputted, the Euclidean distance $d_{n}$ is calculated to evaluate the distance with each row of the memory vector $\mathrm{X}^{n c}$.

$$
\begin{aligned}
& d=\left[\begin{array}{c}
d_{1} \\
d_{2} \\
\vdots \\
d_{N}
\end{array}\right] \\
& d_{n}=\sqrt{\sum_{j=1}^{J}\left(x_{n, j}-x_{j}\right)^{2}}
\end{aligned}
$$

The calculated distance $d_{n}$ is used to evaluate the similarity between the memory vector and the currently measured variable, and a corresponding weight $\omega_{n}$ is given. Gaussian kernel function was used for weight $\omega_{n}$ calculation.

$$
\omega_{n}=\frac{1}{\sqrt{2 \pi} h} e^{-\frac{d_{n}^{2}}{h^{2}}}
$$

Here, $h$ is a parameter that determines the width of the kernel function. When $h$ is higher, a higher weight is assigned to a memory vector which has a higher similarity. Finally, weight $\omega_{n}$ and memory vector $\mathrm{X}^{n c}$ are combined and the estimate $\hat{X}$ is calculated by weighted averaging. 


$$
\begin{aligned}
& \hat{X}=\left[\begin{array}{llll}
\hat{x}_{1} & \hat{x}_{2} & \ldots & \hat{x}_{J}
\end{array}\right] \\
& \hat{x}_{j}=\frac{\sum_{i=1}^{N} \omega_{i} X^{n c}(j, i)}{\sum_{i=1}^{N} \omega_{i}}
\end{aligned}
$$

Figure 3 shows the principles of the early warning system using the AAKR technique. Various variables measured by the instruments of the power plants are collected and estimates are calculated by comparing the measured data with a memory vector based on the historical data. In order to improve the accuracy of the signal estimation, the signals constituting the memory vector are analyzed and grouped based on correlation analysis among them (P. Baradi et al., 2011). And the reliability of the estimate is secured by generating the memory vector of the past 18 months of data in one cycle.

After the estimate is calculated, it is compared with the real-time measurements to obtain the residual and based on the residuals, it is determined whether the equipment is working normally.

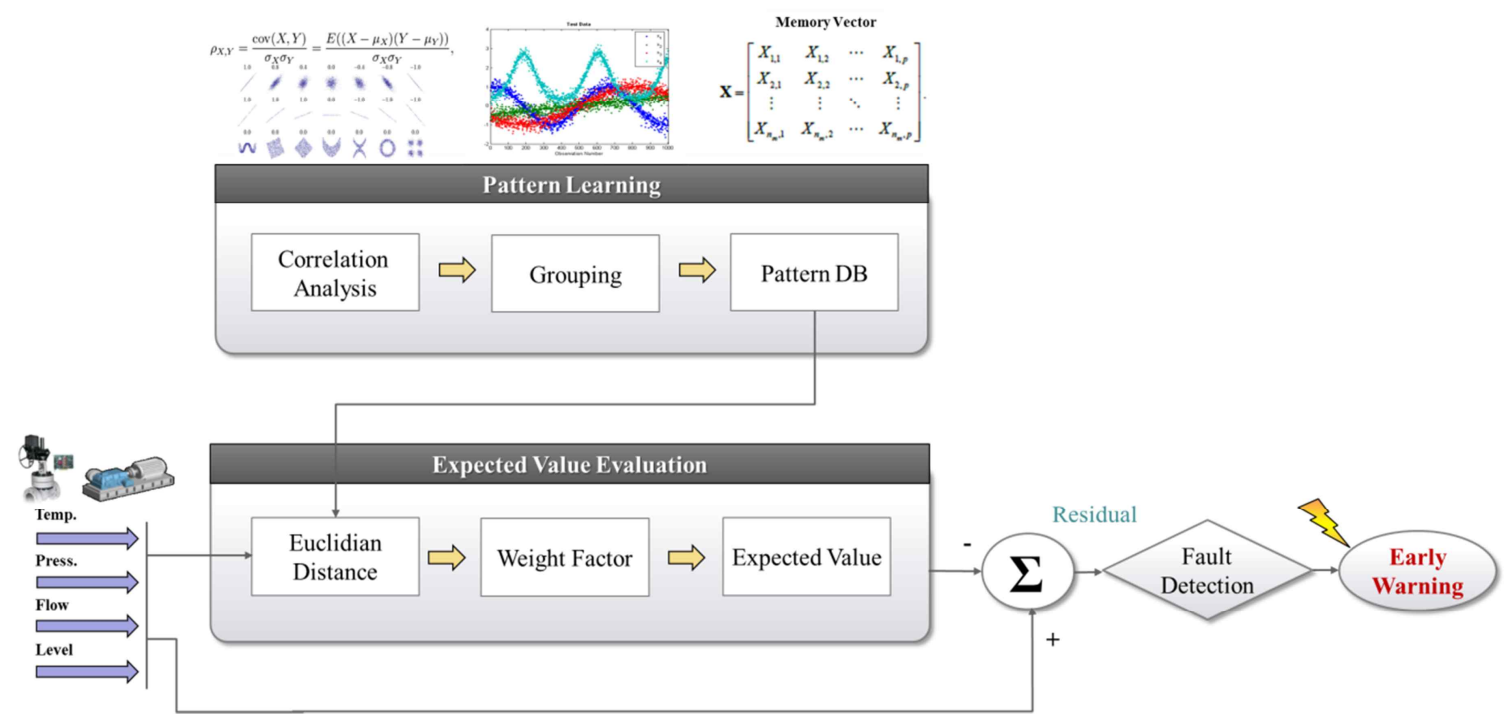

Figure 3. Early Warning System using AAKR Technique

\section{Operation Results of the Early Warning System}

According to the early warning analysis report from June to December in 2016, a total of 6,519 early warning occurred, resulting in about 1.29 alarms per a day for one unit. Of these, 3,016 (46.26\%) cases were predicted alarms from switch operation of equipments or periodic tests of the systems. 1,846 (28.32\%) cases were false alarms caused by inaccuracy of the system and $1,634(25.07 \%)$ cases were judged to valid early warning caused by anomalies in the equipment and measuring instruments.

After comparing the early warning history with the actual power plant maintenance records, we identified that in 2016, a total of 282 cases of equipment anomalies have been successfully detected in advance. Among these, 14 cases have turned out significant anomalies that could have triggered a cutback of the power plant without the alerts from the early warning system. 


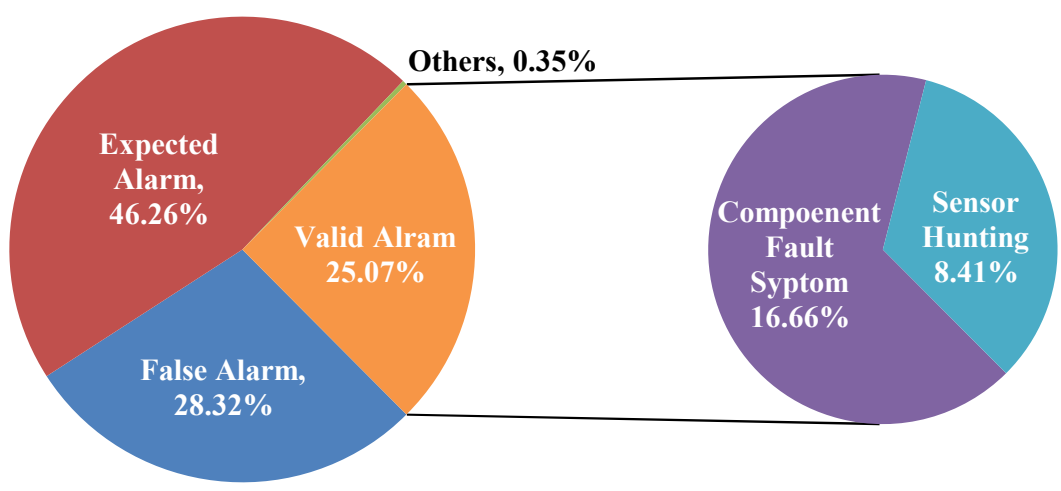

Figure 4. Statistic of Early Warning in $2^{\text {nd }}$ Half of 2016

\section{Case Study}

In this chapter, we are going to present two cases of early warning. In the first case, the early warning system prevented failures by detecting equipment defects in advance. And the other case, alarms were caused by an unusual change in the external environment, and the system went through a pattern relearning to enhance the reliability.

\subsection{Early warning on the MSR temperature transmitter}

Early warning occurred on the temperature transmitter at Stage 2 of the moisture separator reheater(MSR) at 3:07 on October 24,2016 . According to the signal analysis, the temperature which maintained $251^{\circ} \mathrm{C}$ at normal operation decreased in October 11 , and the instantaneous decrease of about $7^{\circ} \mathrm{C}$ compared with the estimated value occurred at the time of the early warning. However, the signals of the temperature transmitter of the other parts remained in the normal range, and any fluctuation did not appear even when the early warning was triggered. Therefore, it was presumed that this early warning was caused by a fault in the measuring instrument. Figure 5 shows the temperature signals at Stage 2 of the MSR, and the round marks indicate the occurrence of early warning.

On October 25, the power plant was notified of the details. Through the maintenance, it was found that the terminal of the temperature transmitter was loosened, affecting the signal transmission. When the issue was resolved, the signals were restored to the normal range as shown in Figure 6.

Table 1. Early Warning of MSR Stage 2 Temperature

\begin{tabular}{|c|c|c|c|}
\hline Timestamp & \multicolumn{3}{|c|}{ 2016-10-24 03:07 } \\
\hline System & $\begin{array}{c}\text { Moisture Separator \& } \\
\text { Reheater }\end{array}$ & TAG & $\begin{array}{c}\text { STG2 Right Down External } \\
\text { Temperature }\end{array}$ \\
\hline Actual & $242.38 \operatorname{deg} \mathrm{C}$ & Expected & $251.38 \mathrm{deg} \mathrm{C}$ \\
\hline
\end{tabular}




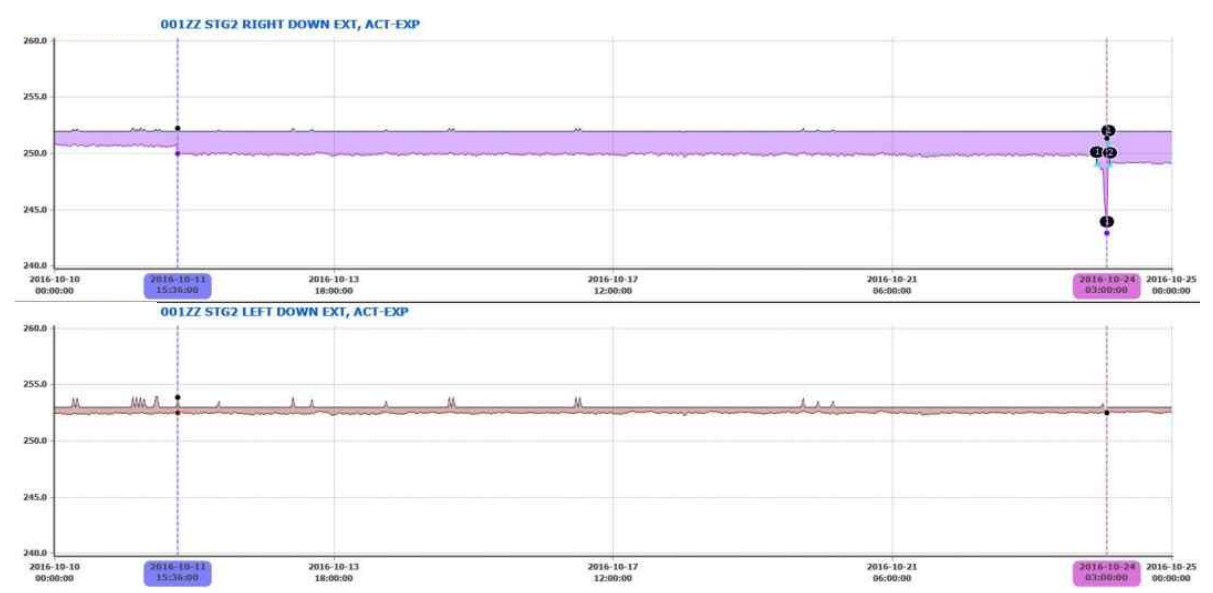

Figure 5. Details of Signals in Case 4.1

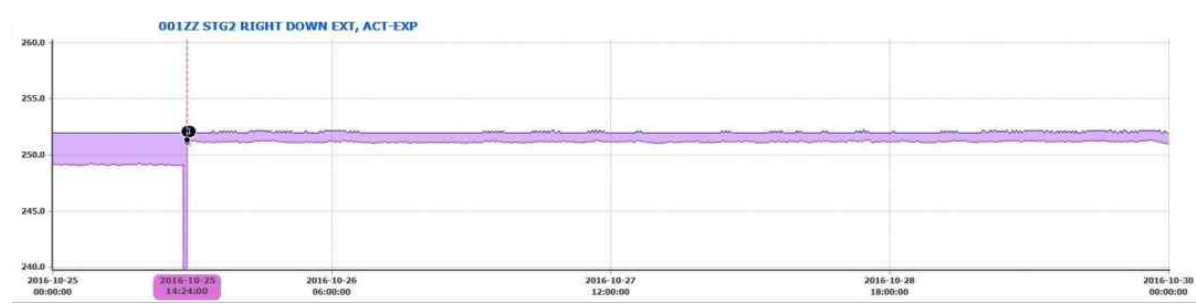

Figure 6. MSR Stage 2 Temperature after Maintenance

\subsection{Case of re-learning from environmental influences}

Figure 7 compares the inlet temperatures of the condenser early August in 2015 with those of in 2016. In the summer of 2016, the temperature of the seawater rose by about 2 degrees compared to the previous year due to an extremely hot weather. Since the seawater is the ultimate heat sink of the nuclear power plant, when the temperature of the seawater rose, huge change occurred in the plant operating data. Since the early warning system generated the pattern model from the operating data in previous cycle, most of the temperature related signals issued early warning, and such influence gradually caused changes in the other signals. Table 2 lists examples of early warning caused by the changes in the seawater temperature. This case is a temporary case where the decline in accuracy of the previous pattern model was caused by an abnormal change in the environment. In order to avoid such false alarms, we changed the fault decision rules for early warnings on the related signals. Conceptually, the early warning occurs when the residual exceeded the threshold as shown in the following formula. Based on expert reviews, the threshold value $r_{\text {thres hou }}$ was temporarily raised to alleviate the fault decision rule and prevent the false alarms.

$$
r=X-\hat{X}>r_{\text {thres holl }}
$$

After the seawater temperature recovered its normal level, the original fault decision rule was restored. In addition, the reliability of the early warning system has been improved by learning additional data of the 
relevant range, thus strengthening the pattern model. This case paradoxically shows the reliability of the system and how it can be enhanced.

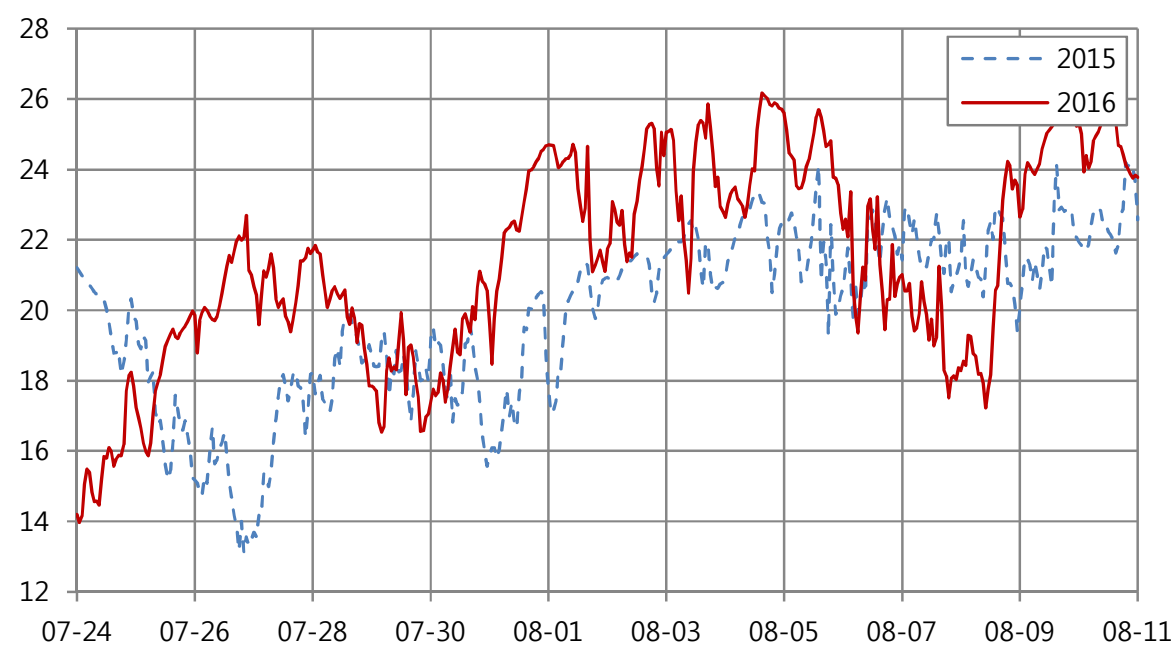

Figure 7. Comparison of Condenser Inlet Water Temperature

Table 2. Examples of Early Warning due to Abnormal Seawater Temperature

\begin{tabular}{|c|l|c|c|c|c|}
\hline \multicolumn{1}{|c|}{ Timestamp } & \multicolumn{1}{|c|}{ Description } & Act. & Exp. & Res. & Unit \\
\hline 2016-07-24 14:55:13 & RCP 1B Upper Thrust Bearing Temp. & 96.95 & 88.20 & 8.75 & $\operatorname{deg} \mathrm{C}$ \\
\hline 2016-07-25 10:02:00 & $\begin{array}{l}\text { Condenser Pump Motor Bearing } \\
\text { Temp. }\end{array}$ & 50.43 & 69.11 & -18.68 & $\operatorname{deg} \mathrm{C}$ \\
\hline 2016-07-27 17:20:00 & SGBD REG HX Outlet Temp. & 52.30 & 53.97 & -1.67 & $\operatorname{deg} \mathrm{C}$ \\
\hline 2016-08-07 15:58:17 & RCP 1A Upper Journal Bearing Temp. & 53.58 & 47.75 & 5.83 & $\operatorname{deg~C}$ \\
\hline 2016-08-09 13:19:50 & MSR A 2nd Stage RHT Pressure & 71.71 & 72.03 & -0.32 & $\mathrm{Kg} / \mathrm{cm}^{2} \mathrm{G}$ \\
\hline 2016-08-09 20:14:35 & Seal Injection HX Discharge Temp. & 43.58 & 38.66 & 4.85 & $\mathrm{deg} \mathrm{C}$ \\
\hline 2016-08-21 05:48:59 & SG 1 Pressure Ch. B & 75.68 & 75.92 & -0.24 & $\mathrm{Kg} / \mathrm{cm}^{2} \mathrm{~A}$ \\
\hline 2016-08-22 17:52:58 & Steam Header 2 Pressure & 72.56 & 72.85 & -0.29 & $\mathrm{Kg} / \mathrm{cm}^{2} \mathrm{G}$ \\
\hline
\end{tabular}

\section{Conclusion}

In this paper, we introduced the functions and roles of the early warning system by presenting how the online monitoring system in the nuclear power plants operates. The early warning system has been applied to 24 plant units since 2016 to detect the abnormality through the difference between the measured values and the estimated values using the AAKR technique. We analyzed the operation result of the system and confirmed that the system was operating properly. Furthermore, the two cases proved the validity and the reliability of the system. 


\section{References}

E. Davis, D. Funk, D. Hooten and R. Rusaw (2000), "On-Line Monitoring of Instrument Channel Performance",EPRI, Palo Alto, CA: 2000. 1000604.

B. Rasmussen, A. Hussey and E. Davis (2004). "Equipment Condition Assessment: Volume 1: Application of On-Line Monitoring Technology", EPRI, Palo Alto, CA: 2004. 1003695

Q. Zhang, M. Basseville and A. Benveniste (1994), "Early Warning of Slight Changes in Systems", Automatica, Vol. 30, Issue 1, pp95-113.

G. Kauermann, M. Marlene and J. C. Raymond (1998) "The efficiency of bias-corrected estimator for nonparametric kernel estimation based on local estimating equations", Statistics and Probability Letters, Vol. 37, pp.41-47.

J.W. Hines, D. Garvey, R. Seibert, and A. Usynin (2008), “Technical Review of On-Line Monitoring Techniques for Performance Assessment: Volume 2" Theoretical Issues", NUREG/CR-6895.

P. Baraldi, R. Canesi, E. Zio, R. Seraoui, R. Chevalier (2011) "Signal Grouping for Condition Monitoring of Nuclear Power Plant Components", Advances in Safety, Reliability and Risk Management: ESREL 2011 